\title{
BOUNDARY BEHAVIOUR OF SOBOLEV MAPPINGS
}

\author{
PIOTR HAJLASZ
}

(Communicated by Theodore W. Gamelin)

\begin{abstract}
Riemann mapping between bounded domains belong to Sobolev space $W^{1,2}$. We investigate the boundary behaviour of Sobolev mappings, and hence it applies to conformal and more general quasiconformal mappings. We generalize a theorem of Øksendal.
\end{abstract}

\section{INTRODUCTION}

If the mapping $f: \bar{B} \rightarrow R^{2}$ is conformal and continuous at the boundary, then the integral $\int_{B}|\nabla f|^{2}$ is finite. Indeed, it follows from Cauchy-Riemann equations that $\left|f_{z}\right|^{2}=J_{f}$, and the integral $\int_{B} J_{f}$ is finite because it is Lebesgue measure of the image. This observation suggests the use of Sobolev function theory in the investigation of boundary behaviour of conformal mappings. Such an approach was successfully applied by Malý and Martio [9]. We will follow the general idea of [9] in connection with pointwise estimates taken from [3].

By $H^{d}$ we denote $d$-dimensional Hausdorff's measure. The average value of $f$ will be denoted by $f_{A}=\mu(A)^{-1} \int_{A} f d \mu=f_{A} f d \mu$. For $1 \leq p<\infty$, the Sobolev space of functions with $p$-integrable gradient is denoted by $W^{1, p}$. By $Q$ we denote an $n$-dimensional cube, and finally by $C$ we denote a general constant which can change its value even in the same proof.

\section{RESULT}

We say that the set $A \subset R^{n}$ is $d$-local if $H^{d}(A)<\infty$ and

$$
\sup _{x \in A, r \leq 1} r^{-d} H^{d}(A \cap B(x, r))<\infty .
$$

If $f \in W^{1, p}(\Omega)$, then we assume that $f$ is defined at every point by the formula $f(x):=\lim \sup _{r \rightarrow 0} f_{B(x, r)} f(z) d z$. It is well known that for $f \in W^{1,1}(Q)$, the inequality $\left|f(x)-f_{Q}\right| \leq C \int_{Q}|\nabla f(z)||x-z|^{1-n} d z$ holds for almost all $x \in Q$

Received by the editors July 6, 1993.

1991 Mathematics Subject Classification. Primary 46E35, 30D40, 30C65.

Key words and phrases. Sobolev mappings, Riemann mapping, quasiconformal mappings, trace, boundary behaviour, maximal function, Hausdorff measure.

The work was supported by a KBN grant no. 210579101 . 
(see [4, Lemma 7.16]). Now applying the limiting process (used in the definition of $f$ at every point) we get that this inequality holds everywhere (maybe with a worse constant $C$; see [5] or [2] for details). By $W^{1, p}\left(\Omega, R^{n}\right)$ we denote the space of mappings $f=\left(f_{1}, \ldots, f_{n}\right): \Omega \rightarrow R^{n}$ such that all coordinate functions $f_{i}$ belong to $W^{1, p}(\Omega)$. In the case of mappings we also assume that $f$ is defined at every point. Namely, we apply the above procedure to coordinate functions of $f$.

Theorem 1. Let $f \in W^{1, p}\left(\Omega, R^{n}\right)$, where $\Omega \subset R^{n}$ is an open set and $1 \leq$ $p<\infty$, be defined at every point as above. Let $A \subset \Omega$ be a $d$-local set where $p+d>n$. Then there exists $E \subset A$ with $H^{d}(E)=0$ such that for $0<\alpha \leq d$ and $\beta \geq \frac{\alpha p}{p+d-n}$, the following implication holds:

$$
F \subset A \backslash E, H^{\alpha}(F)<\infty \Rightarrow H^{\beta}(f(F))=0 .
$$

If $\Omega$ is a bounded domain with Lipschitz boundary and $f \in W^{1, p}(\Omega)$, then we define $f$ at the boundary points by $f(x):=\limsup _{r \rightarrow 0} f_{B(x, r) \cap \Omega} f(z) d z$ for all $x \in \partial \Omega$. Since we have an extension operator for such domains, we can apply the above theorem to a $d$-local set $A \subset \partial \Omega$.

Corollary 1. Let $\Omega$ be a bounded domain with Lipschitz boundary. If $f \in$ $W^{1, n}\left(\Omega, R^{n}\right)$ is defined at every point of $\bar{\Omega}$ as above, then there exists $E \subset \partial \Omega$ with $H^{n-1}(E)=0$ such that for $\alpha \leq n-1$, the following implication holds:

$$
F \subset \partial \Omega \backslash E, H^{\alpha}(F)<\infty \Rightarrow H^{\alpha n /(n-1)}(f(F))=0 .
$$

If we put $\alpha=n-1$, then the above corollary is a very special case of [9, Corollary $\mathrm{H}$ ] (namely, they have proved that in this case one can take $E$ with Hausdorff's dimension equal to zero); however, the results of [9] do not apply to other cases.

As we mentioned in the Introduction, the above corollary for $n=2$ applies to conformal mappings. In this case it was proved with $\alpha=1$ (for conformal mappings of course) by Øksendal [10]. The conformal case follows also from much more delicate estimations of Jones and Makarov [8]. In the case of plane quasiconformal mappings Corollary 1 was proved for $\alpha=1$ by Heinonen and Martio [7].

It seems that the above corollary with $n \geq 3, \alpha<n-1$ is new even for quasiconformal mappings (compare the discussion in [9]).

Since the theorem has a local nature, we can assume that $\Omega=R^{n}$. In the proof we need some lemmas. Let $M_{R}^{\lambda} g(x)=\sup _{r<R} r^{\lambda} f_{B(x, r)}|g(z)| d z$.

Lemma 1. If $f \in W^{1, p}\left(R^{n}\right)$ and $0<\lambda<1$, then the following inequality holds everywhere:

$$
|f(x)-f(y)| \leq C|x-y|^{1-\lambda}\left(M_{|x-y|}^{\lambda}|\nabla f|(x)+M_{|x-y|}^{\lambda}|\nabla f|(y)\right) .
$$

Remark. This inequality follows from more general results of [3]. It is also related to the inequalities in $[1,5,2]$, but we will prove it for the sake of completeness.

In the proof of Lemma 1 we need the following Hedberg's type lemma (compare $[6,12,2,3])$. 
Lemma 2. If $\gamma>\lambda \geq 0$, then there exists a constant $C$ such that

$$
\int_{Q} \frac{|g(z)|}{|x-z|^{n-\gamma}} d z \leq C(\operatorname{diam} Q)^{\gamma-\lambda} M_{\text {diam } Q}^{\lambda} g(x)
$$

for all $g \in L^{1}(Q)$ and all $x \in Q$.

Proof. We break the integral on the left-hand side into the sum of the integrals over 'rings' $Q \cap\left(B\left(x, \operatorname{diam} Q / 2^{k}\right) \backslash B\left(x, \operatorname{diam} Q / 2^{k+1}\right)\right)$. In each 'ring' we have $|x-z|^{\gamma-n} \approx\left(\operatorname{diam} Q / 2^{k}\right)^{\gamma-n}$. Now we estimate the integral over the 'ring' by the integral over the ball $B\left(x, \operatorname{diam} Q / 2^{k}\right)$, and the lemma follows easily.

Proof of Lemma 1. Let $x, y \in Q, \operatorname{diam} Q \approx|x-y|$. We have

$$
\begin{aligned}
|f(x)-f(y)| & \leq\left|f(x)-f_{Q}\right|+\left|f(y)-f_{Q}\right| \\
& \leq C\left(\int_{Q} \frac{|\nabla f(z)|}{|x-z|^{n-1}} d z+\int_{Q} \frac{|\nabla f(z)|}{|y-z|^{n-1}} d z\right) \\
& \leq C|x-y|^{1-\lambda}\left(M_{|x-y|}^{\lambda}|\nabla f|(x)+M_{|x-y|}^{\lambda}|\nabla f|(y)\right) .
\end{aligned}
$$

The last inequality follows from Lemma 2 with $\gamma=1$.

Lemma 3. If $A \subset R^{n}$ is $d$-local and $\lambda=\frac{n-d}{p}$, then there exists a constant $C$ such that

$$
H^{d}\left(\left\{x \in A \mid M_{1}^{\lambda} g(x)>t\right\}\right)<\frac{C}{t^{p}} \int_{R^{n}}|g(z)|^{p} d z
$$

for all $g \in L^{p}\left(R^{n}\right)$.

Lemma 3 states that the operator $M_{1}^{\lambda}$ is of weak type $(p, p)$ (between $L^{p}\left(R^{n}\right)=L^{p}\left(R^{n}, H^{n}\right)$ and $L^{p}\left(A, H^{d}\right)$ ( $H^{s}$ indicates the measure with respect to which we define the $L^{p}$ norm)). The proof follows by a standard application of Vitali's type covering lemma, in the same way as in the classical Hardy-Littlewood's maximal theorem (compare [11, Chapter 1]).

Lemma 4. Let $A$ be a d-local set, $d<n, g \in L^{p}\left(R^{n}\right)$, and $\lambda=\frac{n-d}{p}$. Then to every $\varepsilon>0$ there exists $E \subset A$ with $H^{d}(E)<\varepsilon$ such that $M_{r}^{\lambda} g(x) \stackrel{r=0}{\longrightarrow} 0$ uniformly with respect to $x \in A \backslash E$.

Proof. Let $k \in C_{0}^{\infty}\left(R^{n}\right)$ be such that $\|k-g\|_{L^{p}}^{p}<\varepsilon^{p+1}$. Let $h=k-g$. Evidently there exists $R<1$ with $\sup _{x \in R^{n}} M_{R}^{\lambda} k<\varepsilon$ (because $k \in C_{0}^{\infty}$ ). Now since $M_{R}^{\lambda} g \leq M_{1}^{\lambda} h+M_{R}^{\lambda} k$,

$$
H^{d}\left(\left\{M_{R}^{\lambda} g>2 \varepsilon\right\}\right) \leq H^{d}\left(\left\{M_{1}^{\lambda} h>\varepsilon\right\}\right) \leq \frac{C}{\varepsilon^{p}} \int_{R^{n}}|h|^{p}<C \varepsilon .
$$

Let $R_{i}, \varepsilon_{i} \rightarrow 0$ be such that $H^{d}\left(\left\{M_{R_{i}}^{\lambda} g>\varepsilon_{i}\right\}\right)<\varepsilon / 2^{i}$. Now it suffices to put $E=\bigcup_{i}\left\{M_{R_{i}}^{\lambda} g>\varepsilon_{i}\right\}$.

Proof of Theorem 1. We prove that Theorem 1 holds with $E=\bigcap_{i} E_{i}$, where $E_{i}$ is as in Lemma 4 with $g=|\nabla f|$ and $\varepsilon=1 / i$. It suffices to prove that the following implication holds for all $i$ :

$$
F \subset A \backslash E_{i}, H^{\alpha}(F)<\infty \Rightarrow H^{\beta}(f(F))=0 .
$$


Let $F \subset \bigcup_{i} B\left(x_{i}, r_{i}\right), x_{i} \in F, \sum r_{i}^{\alpha}<C H^{\alpha}(F), \sup _{i} r_{i}<\varepsilon<1$. Since $f$ is $(1-\lambda)$-Hölder continuous on $A \backslash E_{i}$ with $\lambda=\frac{n-d}{p}$, then $f\left(B\left(x_{i}, r_{i}\right) \cap F\right) \subset$ $B\left(f\left(x_{i}\right), s_{i}\right)$ where

$$
s_{i}<C\left(\sup _{F} M_{\varepsilon}^{\lambda}|\nabla f|\right) r_{i}^{1-\lambda} .
$$

Hence $H^{\beta}(f(F))=0$ because according to Lemma 4

$$
\sum_{i} s_{i}^{\beta} \leq\left(\sup _{F} M_{\varepsilon}^{\lambda}|\nabla f|\right)^{\beta} \sum_{i} r_{i}^{\alpha} \stackrel{\varepsilon=0}{\rightarrow} 0
$$

\section{ACKNOWLEDGMENT}

I am indebted to Professor Olli Martio for pointing my attention to a problem and very fruitful discussions. I am also indebted to Pawel Strzelecki for a valuable idea which has led to appearance of this paper.

\section{BIBLIOGRAPHY}

1. B. Bojarski, Remarks on some geometric properties of Sobolev mappings, Functional Analysis \& Related Topics (Shozo Koshi, ed.), World Scientific, Singapore, 1991.

2. B. Bojarski and P. Hajlasz, Pointwise inequalities for Sobolev functions and some applications, Studia Math. 106 (1993), 77-92.

3. _ Pointwise inequalities for Sobolev functions and some applications, II (in preparation).

4. D. Gilbarg and N. Trudinger, Elliptic partial differential equations of second order, SpringerVerlag, New York, 1983.

5. P. Hajłasz, Change of variables formula under minimal assumptions, Colloq. Math. 64 (1993), 93-101.

6. L. Hedberg, On certain convolution inequalities, Proc. Amer. Math. Soc. 36 (1972), 505-510.

7. J. Heinonen and O. Martio, Estimates for F-harmonic measures and Øksendal's theorem for quasiconformal mappings, Indiana Univ. Math. J. 36 (1987), 659-683.

8. P. Jones and N. G. Makarov, Density properties of harmonic measure, preprint.

9. J. Malý and O. Martio, Lusin's condition $(N)$ and mappings of the class $W^{1, n}$, J. Reine Angew. Math. (to appear).

10. B. Øksendal, Null sets for measures orthogonal to $R(X)$, Amer. J. Math. 94 (1972), 331-342.

11. E. Stein, Singular integrals and differentiability properties of functions, Princeton Univ. Press, Princeton, NJ, 1970.

12. W. Ziemer, Weakly differentiable functions, Springer-Verlag, New York, 1989.

Instytut Matematyki, Uniwersytet WaRszawski, Ul. Banacha 2, 02-097 WaRsZawa, PolSKA

E-mail address: HAJLASZOMIMUW.EDU.PL 